\title{
Bortezomib-Based Chemotherapy with Autologous Stem Cell Transplantation for Monoclonal Gammopathy of Renal Significance: A Case Report and Literature Review
}

\author{
Jing Huang ${ }^{a}$ Chunyan Sun $^{b} \quad$ Hua Su $^{a}$ Chun Zhang ${ }^{a}$ Jing Xiong ${ }^{a}$ \\ ${ }^{a}$ Department of Nephrology, Union Hospital, Tongji Medical College, Huazhong University \\ of Science and Technology, Wuhan, China; ${ }^{b}$ Department of Hematology, Union Hospital, \\ Tongji Medical College, Huazhong University of Science and Technology, Wuhan, China
}

\section{Keywords}

Bortezomib · Autologous stem cell transplantation - Monoclonal gammopathy of renal significance

\begin{abstract}
Background/Aims: The term monoclonal gammopathy of renal significance (MGRS) was introduced in 2012 to emphasize kidney lesions in monoclonal gammopathy patients. Bortezomib-based chemotherapy has become the first-line treatment for MGRS. Objectives: The objective of this study was to investigate whether the strategy of combining chemotherapy with autologous stem cell transplantation (ASCT) could improve prognosis and decrease functional kidney impairment in patients with MGRS. Methods: We reported the case of a 44-year-old Asian patient who was diagnosed with MGRS and received 5 cycles of Velcade ${ }^{\circledR}$ (a trade name for bortezomib), thalidomide, and dexamethasone therapy (VTD therapy), and subsequently underwent ASCT. In addition, we performed a literature review and summarized the latest advances in the characterization, treatment, and prognosis of MGRS. Results: The patient was diagnosed with light chain deposition disease by renal biopsy. After 5 cycles of VTD therapy, the patient had a very good partial response characterized by the resolution of M-protein (20.2\% before treatment vs. $2.5 \%$ after treatment), remission of the level of serum free lambda (FLAM; over $80 \%$ decline), and normalization of the serum free light chain (sFLC) ratio ( $\kappa$ to $\lambda$ ). He also had a renal response characterized by a decreased serum creatinine level (1.61 vs. $1.34 \mathrm{mg} / \mathrm{dL}$ ) and less severe proteinuria ( $6.77 \mathrm{~g} / 24 \mathrm{~h}$ vs. $1.264 \mathrm{~g} / 24 \mathrm{~h}$ ) after chemotherapy. Importantly, after ASCT, the patient achieved a complete response (CR) characterized by a negative serum immunofixation electrophoresis (IFE) result and a dramatic decrement in FLAM (over 90\%). Furthermore, 6 months after ASCT, the patient still remained in
\end{abstract}




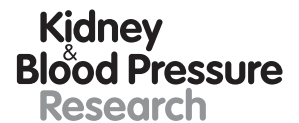

Research

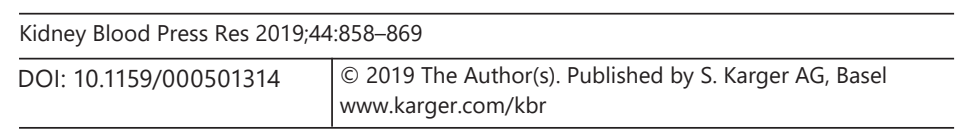

Huang et al.: Bortezomib/Autologous Transplant for Monoclonal Gammopathy of Renal Significance

stable condition with a negative IFE result, normal sFLC ratio, and low level of serum creatinine $(1.31 \mathrm{mg} / \mathrm{dL})$ and proteinuria $(0.339 \mathrm{~g} / 24 \mathrm{~h})$. In our retrospective literature analysis, we found that MGRS patient survival time and renal outcome had been markedly improved by current therapies due to the popularization of bortezomib-based chemotherapy and ASCT. Conclusions: The patient successfully achieved CR after VTD therapy followed by ASCT. However, this treatment is controversial, and a standard therapy recommendation for MGRS has not been established. Bortezomib-based chemotherapy combined with ASCT may have prospects for the treatment of MGRS, but the exact effects of ASCT remain unclear and should be thoroughly investigated.

\section{Introduction}

The clinical term monoclonal gammopathy of renal significance (MGRS) was first delineated by the International Kidney and Monoclonal Gammopathy Research Group in 2012 [1]. The definition of the group of hemato-nephrological entities as MGRS was intended to distinguish the disorders from monoclonal gammopathy of undetermined significance with respect to the risk of severe consequences in the kidney [2]. MGRS entities are characterized by pathogenesis driven by monoclonal immunoglobulin (MIg) secreted by small B cell and plasma cell clones in the absence of overt hematologic malignancy [3]. Patients with MGRS do not meet the criteria for overt multiple myeloma (MM) or systemic lymphoma [4]. Therefore, some kidney diseases that result from high-grade lymphoproliferative disorders or are independent of MIg, such as those resulting from drug toxicity or metabolic disorder, are excluded from MGRS [5]. Meanwhile, light chain deposition disease (LCDD), which includes only light chains (LCs), is the most common form of MGRS [6].

Renal involvement caused by MGRS is severe and irreversible, and usually presents as atypical manifestations, such as hypertension, nephrotic syndrome, and renal insufficiency, that, if not successfully treated, evolve to end-stage renal disease (ESRD) [7-9]. In addition, MGRS is associated with high mortality, poor patient survival, and unsatisfactory renal outcomes with a high rate of recurrence even after triplet chemotherapy, autologous stem cell transplantation (ASCT), or renal transplantation [1-5, 8]. However, the prevalence of MGRS has consistently been underestimated as rare conditions. Currently, there is no established treatment for MGRS, and only limited clinical trials are available [10]. Herein, we report the case of a patient with IgA- $\lambda$ subtype primary LCDD (PLCDD) and summarize the current status of the characterization, treatment, and prognosis of MGRS, with the aims of improving public awareness and shedding light on the treatment of MGRS.

\section{Case Report}

A 44-year-old man without any previous medical history came to our hospital with lower limb edema on March 22, 2017. Laboratory findings showed severe proteinuria (24-h urinary test: $6.077 \mathrm{~g} / 24 \mathrm{~h}$ ), hypoproteinemia (serum albumin: $2.59 \mathrm{~g} / \mathrm{dL}$ ), renal insufficiency (estimated glomerular filtration rate [eGFR]: $51 \mathrm{~mL} / \mathrm{min} / 1.73 \mathrm{~m}^{2}$, estimated with the Chronic Kidney Disease Epidemiology Collaboration [CKD-EPI] formula), elevated serum creatinine (1.61 mg/dL), and mild anemia (hemoglobin: $10.7 \mathrm{~g} / \mathrm{dL}$ ). First, we strongly suspected nephrotic syndrome and immediately performed renal biopsy on the patient.

Light microscopy showed many hypocellular nodules in the mesangium with a large, pathologic glomerulus and severe expansion of the mesangial area. The biopsy was 


\section{Kidney \\ Blood Pressure Research}

\begin{tabular}{l|l}
\hline Kidney Blood Press Res 2019;44:858-869 \\
\hline DOI: 10.1159/000501314 & $\begin{array}{l}\text { @ 2019 The Author(s). Published by S. Karger AG, Basel } \\
\text { www.karger.com/kbr }\end{array}$ \\
\hline
\end{tabular}

Huang et al:: Bortezomib/Autologous Transplant for Monoclonal Gammopathy of Renal Significance
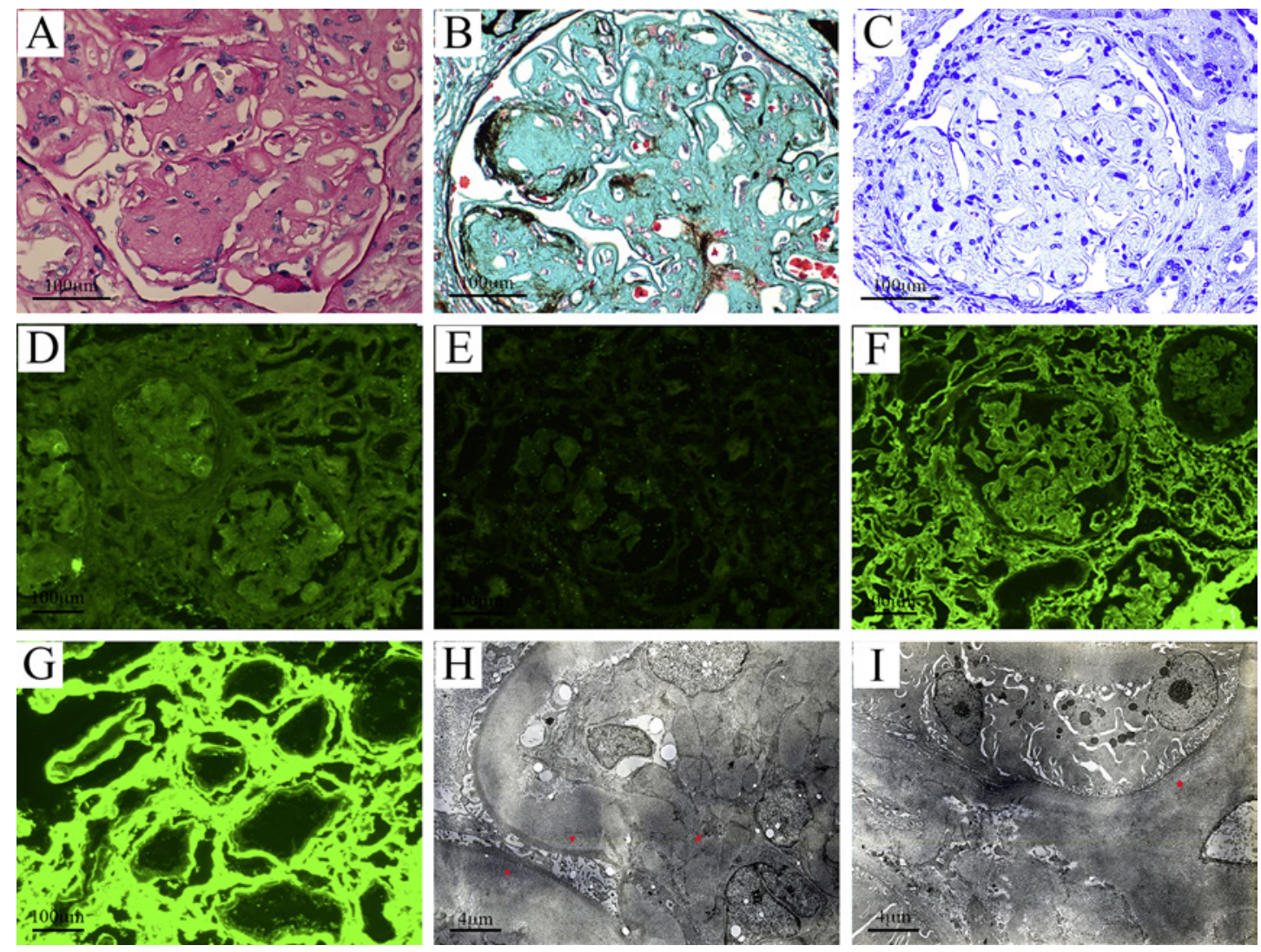

Fig. 1. The pathological findings of this patient by renal biopsy. A, B Hypocellular nodules in the glomeruli by $(\mathbf{A})$ periodic acid-Schiff and $(\mathbf{B})$ trichrome staining (both at $\times 600$ ). $\mathbf{C}$ Negative Congo red staining in the kidney biopsy $(\times 600)$. D Negative IgA staining by IF $(\times 400)$. E Negative staining for $\kappa-\operatorname{LC}$ by IF $(\times 400)$. F, G Linear peripheral glomerular capillary wall staining and staining along the TBM. Direct IF for $\lambda$-LC $(\times 400)$. $\mathbf{H}$ Subendothelial, continuous, sedimented, or punctate material along the peripheral capillary wall (arrowheads) and deposition in the mesangium (arrows). I Continuous, sedimented or punctate material along the TBM (pentagram). Transmission EM $(\times 10,000)$.

negative for Congo red staining. Immunofluorescence staining (IF) for $\lambda$-LCs was strongly positive along the glomerular capillary walls, tubular basement membrane (TBM), and tubulointerstitium, whereas $\kappa$-LCs and other antisera (i.e., IgG, IgG1, IgG2, IgG3, IgG4, IgM, IgA, C3, C4, and C1q) were all negative. Electron microscopy (EM) revealed the deposition of some electron-dense granules in the mesangial area, glomerular basement membrane (GBM), TBM, and interstitium (Fig. 1). Serum and urine immunofixation electrophoresis (IFE) suggested IgA- $\lambda$-related monoclonal gammopathy (Fig. 2). IgA was detected at levels up to $13.4 \mathrm{~g} / \mathrm{L}$ (normal range: $0.70-4.00 \mathrm{~g} / \mathrm{L}$ ) and the proportion of M-protein reached $21.8 \%$ of antibodies. Serum free kappa (FKAP) was detected at 19.5 $\mathrm{mg} / \mathrm{L}$ (normal range: $<3.3-19.4 \mathrm{mg} / \mathrm{L}$ ), whereas the serum free lambda (FLAM) was 150.4 $\mathrm{mg} / \mathrm{L}$ (normal range: $<5.71-26.3 \mathrm{mg} / \mathrm{L}$ ) and the ratio of FKAP to FLAM was 0.13 (normal range: $<0.26-1.65)$.

Bone marrow aspirate and biopsy showed an elevated proportion of plasma cells (under $5 \%$ ) with no evidence of amyloid deposition. The bone marrow immunophenotyping assay revealed an abnormal monoclonal plasma cell phenotype $(0.91 \%$ of all nucleated cells), characterized by the expression of CD138, CD 45, and $\lambda$-LC and the absence of CD19, CD56, and 
Kidney

Blood Pressure

Research

\begin{tabular}{|c|c|}
\hline Kidney Blood Press Res 201 & $1: 858-869$ \\
\hline DOI: $10.1159 / 000501314$ & $\begin{array}{l}\text { (c) } 2019 \text { The Author(s). Published by S. Karger AG, Basel } \\
\text { www.karger.com/kbr }\end{array}$ \\
\hline
\end{tabular}

Huang et al:: Bortezomib/Autologous Transplant for Monoclonal Gammopathy of Renal Significance
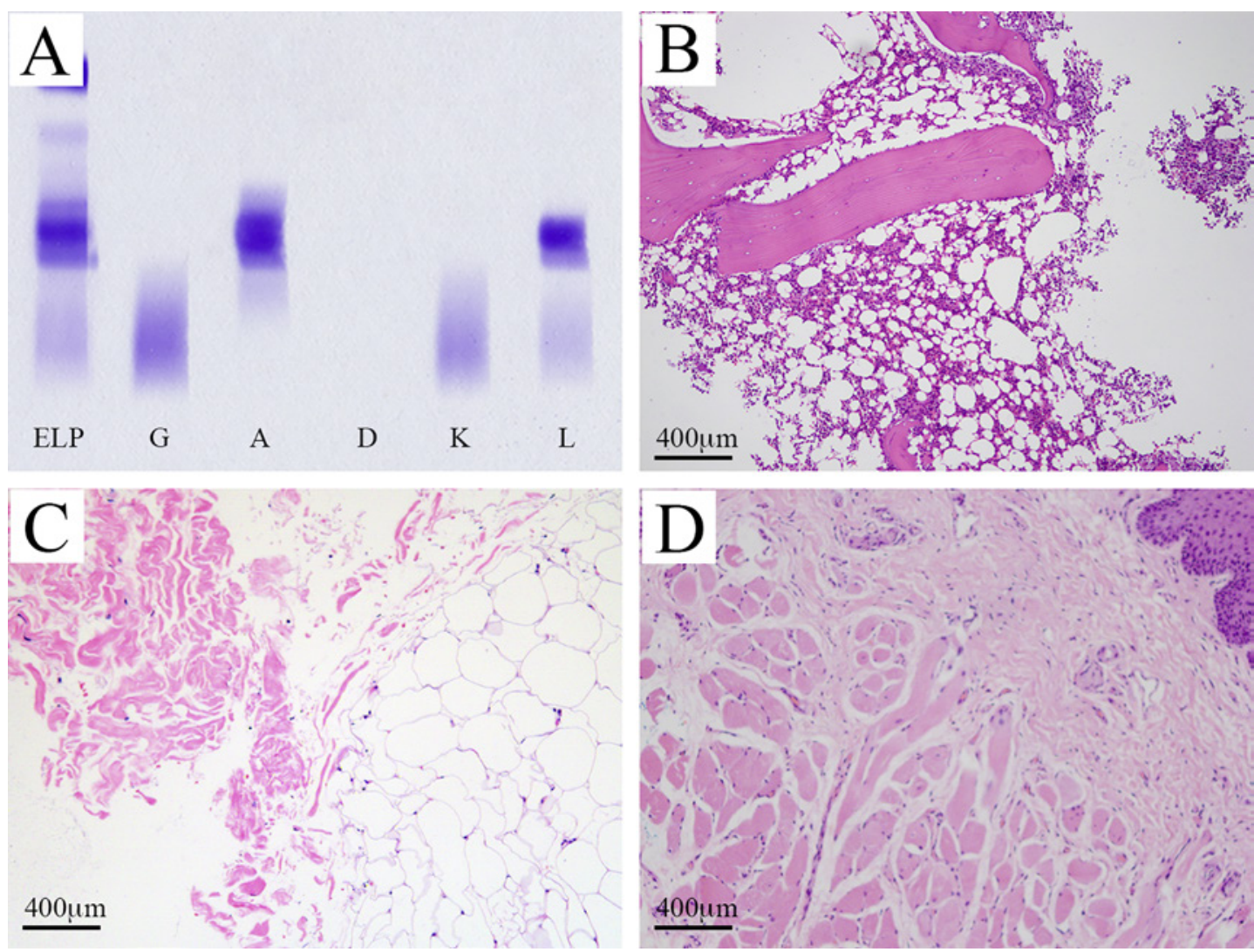

Fig. 2. The hematologic examinations of this patient. A M-protein band that combined with anti-IgA and anti-LC to form a specific deposition band on serum IFE. B Monoclonal proliferation of plasma cells (under $5 \%)$ and a lack of Congo red staining in the bone marrow aspirate and biopsy $(\times 100)$. C Negative Congo red staining in the fat biopsy $(\times 100)$. D Negative Congo red staining in the hyoid tissue biopsy $(\times 100)$.

$\kappa$-LC. However, the chromosome karyotype analysis and MM fluorescence in situ hybridization assay of bone marrow cells proved to be normal.

The patient agreed to hyoid tissue biopsy and fat biopsy, both of which were negative (Fig. 2). All imaging examinations (including skeletal survey, single-electron positron emission tomography, abdominal ultrasound, echocardiogram ultrasound, and cardiac magnetic resonance imaging) were normal. After combining these findings with the examination results, the final diagnosis was PLCDD.

To reduce LC burden and improve renal function, we commenced chemotherapy with Velcade ${ }^{\circledR}$ (a trade name for bortezomib), thalidomide, and dexamethasone (VTD therapy) in May 2017. Thalidomide was orally administered at the initial therapeutic dose of 100 $\mathrm{mg}$ /day for 1 cycle, then adjusted to $75 \mathrm{mg}$ /day for the remaining 4 cycles due to astriction. Dexamethasone $(20 \mathrm{mg}$ ) was administered by subcutaneous injection on days $1,2,4,5,8$, 9,11 , and 12 . Velcade $(2.5 \mathrm{mg}$ ) was administered by intravenous injection on days $1,4,8$, and 11 (Fig. 3). A course of treatment lasted for 12 days, and the next course began at least 9 days later. After undergoing 5 cycles of the triple chemotherapy, the patient achieved a hematologic very good partial response (VGPR) characterized by the resolution of M-protein and normalization of the serum free light chain (sFLC) ratio ( $\kappa$ to $\lambda$ ). He also achieved a good renal response with lower serum creatinine and proteinuria than in the initial stage (Fig. 4). Categorization of the hematologic response was adapted from the 2012 International 
Fig. 3. VTD therapy regimen for this patient. Thalidomide was orally administered at $100 \mathrm{mg} /$ day for 1 cycle and at $75 \mathrm{mg} /$ day for the remaining cycles. Dexamethasone $(20 \mathrm{mg}$ ) was administered to the patient by subcutaneous injection on days $1,2,4,5,8,9,11$, and 12. Velcade ${ }^{\circledR}(2.5 \mathrm{mg})$ was administered by intravenous injection on days $1,4,8$, and 11. A single course of treatment lasted 12 days.

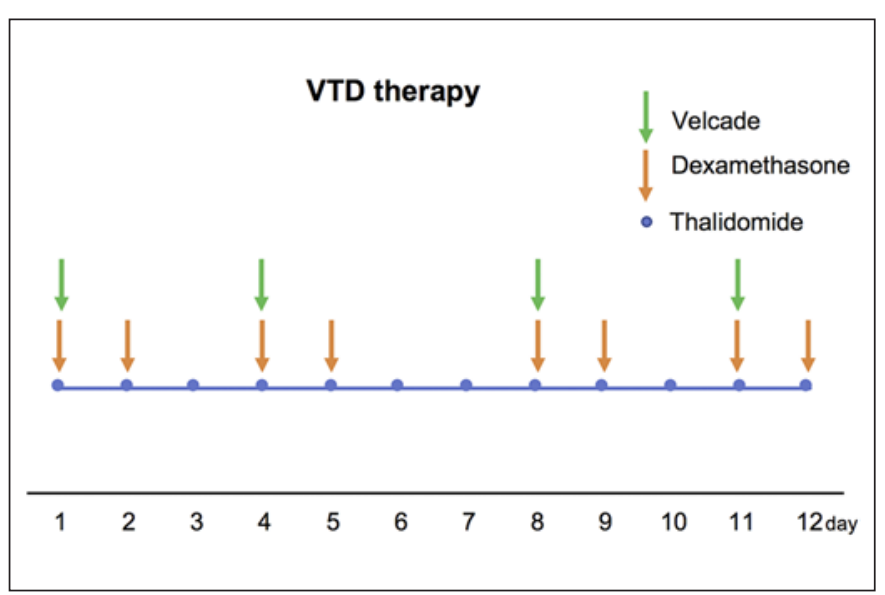

A

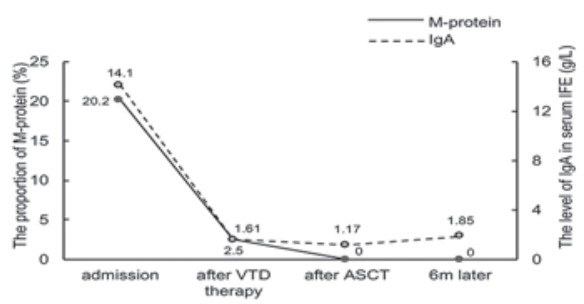

C

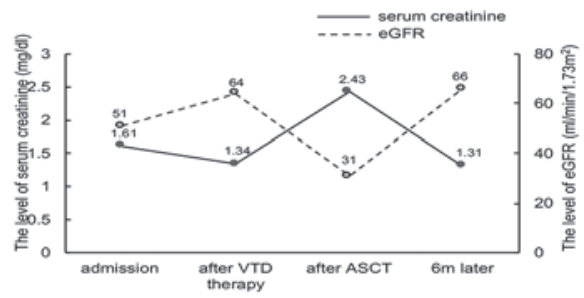

E

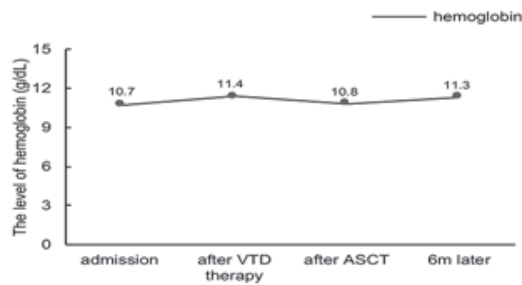

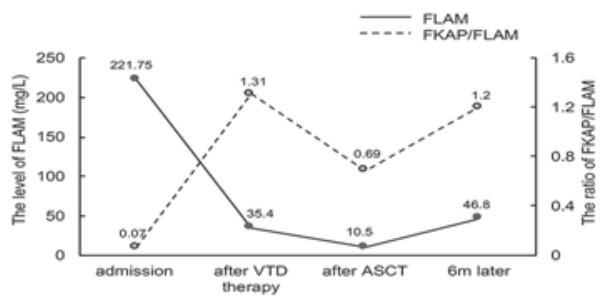

D

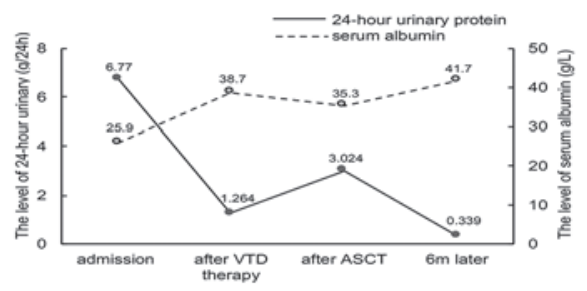

Fig. 4. The dynamic changes in various parameters at different treatment stages of this patient. A Changes in M-protein and IgA, both of which returned to normal after ASCT by serum IFE. B Changes in serum FLAM and the ratio of $\kappa$ to $\lambda$ as assessed by sFLC assay. C Changes in the levels of serum creatinine and eGFR (estimated by CKD-EPI formula). D Changes in the levels of 24-h proteinuria and serum albumin. $\mathbf{E}$ Changes in the levels of hemoglobin. After VTD therapy, 1 month after 5 cycles of VTD therapy; after ASCT, 1 week after ASCT; 6m later, 6 months after ASCT. 
Huang et al.: Bortezomib/Autologous Transplant for Monoclonal Gammopathy of Renal Significance

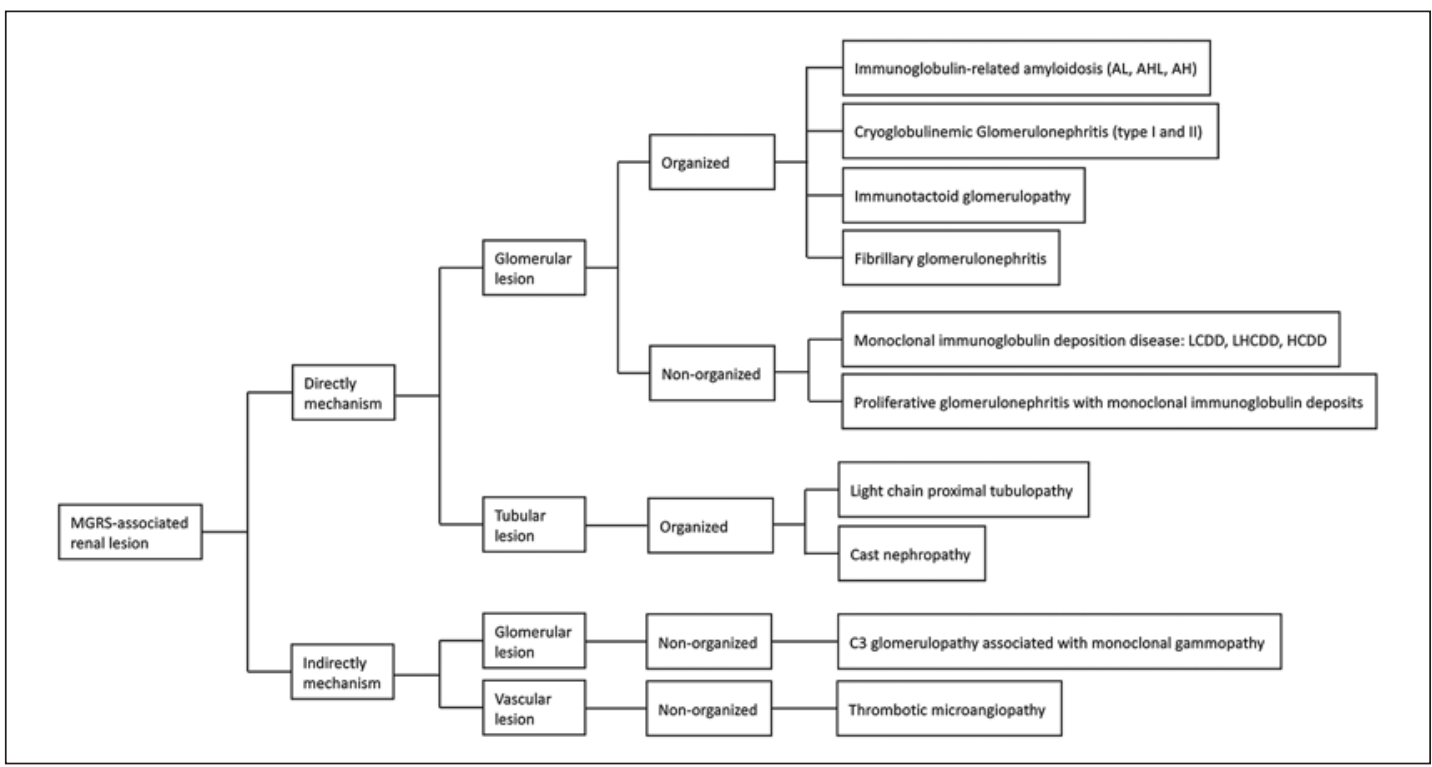

Fig. 5. Diagram of MGRS-associated renal lesions. MGRS, monoclonal gammopathy of renal significance; $A H$, immunoglobulin heavy chain amyloidosis; AHL, immunoglobulin heavy and light chain amyloidosis; AL, immunoglobulin light chain amyloidosis; LCDD, light chain deposition disease; LHCDD, light and heavy chain deposition disease; HCDD, heavy chain deposition disease.

Society of Amyloidosis criteria [11] and the renal response was assessed based on Kidney Disease: Improving Global Outcomes practice guidelines on glomerulonephritis [12]. After his good health was confirmed, the patient underwent ASCT in November 2017. Strikingly, after combined treatment with chemotherapy and ASCT, the patient achieved a complete response (CR) with a negative IFE result (no detectable M-protein or IgA) and marked remission of abnormal FLAM (over 90\% decline compared to pre-treatment) as well as a normal sFLC ratio (Fig. 4). However, an episode occurred 1 week after ASCT, in which some clinical parameters, including creatinine, eGFR (estimated by CKD-EPI formula), hemoglobin, albumin, and proteinuria, slightly deteriorated (Fig. 4C-E). The effects, which are common in posttransplant patients, may have been induced by the ASCT pre-treatment or treatment regimens. We then carried out follow-up observation of this patient. Thalidomide was discontinued when the patient had sustained his response for 3 months after ASCT, and only Velcade and dexamethasone were used in the course of chemotherapy. Six months after ASCT, the patient was still in stable condition with negative IFE findings and a normal $\kappa$-to- $\lambda$ ratio. He also sustained relatively low serum creatinine and 24-h urine protein levels (Fig. 4).

\section{Review of the Literature}

The term MGRS was introduced in 2012 to differentiate the included entities from monoclonal gammopathy of undetermined significance based on the presence of significant kidney lesions; in 2016, MGRS was classified into two groups by Sethi et al. [13] according to pathogenesis. One group is characterized by direct deposition of MIg in the kidney, whereas the other is subject to an indirect mechanism of MIg regulation and activation of the alternative complement pathway (Fig. 5). However, while the designation MGRS helps highlight patients 
Huang et al.: Bortezomib/Autologous Transplant for Monoclonal Gammopathy of Renal Significance

with renal diseases secondary to M-protein secretion by a premalignant clone, it is not a disease or diagnosis in itself $[1,2,7,8]$.

A diagnosis of MGRS usually depends on the detection of MIg $[14,15]$. Detection by IF and EM in the biopsy is indispensable. Immunoelectron microscopy may play an important role in some early cases with atypical pathologic characteristics. Several MIg assays are available in the clinic, including protein electrophoresis (PEP), IFE, and sFLC assays for serum and urine. The sensitivity of the sFLC assay to detect MGRS is higher than those of IFE and PEP [5, 16]. Recently, 2 new tests with higher sensitivities and specificities than sFLC assays have attracted attention. In the time-of-flight technique, a mass spectrometer can quickly and accurately detect $\mathrm{M}$-proteins based on their mass-to-charge ratio. The other new technique, the analysis of urinary exosomes, is a useful tool for the study of cellular proteomics in the urinary tract. Urinary exosomes can be an excellent biomarker for renal response in LCDD [17]. However, the two techniques are not universally used in the clinic. Currently, no single test can provide all of the information necessary to the clinician. A thorough understanding of the capabilities and limitations of these tests will lead to better patient care.

In the past, some research suggested that it was unnecessary for patients with MGRS (except those with immunoglobulin LC amyloidosis [AL amyloidosis]) to receive systematic treatment due to low tumor burden [18]. However, increasing evidence indicates that aggressive therapy to control MIg in MGRS patients could improve renal function, delay the progression to ESRD, and reduce the rate of recurrence after renal transplantation [19]. If left untreated, nonmalignant B cell clones could lead to organ dysfunction and even deteriorate to MM as the disease progresses [20]. Therefore, active treatment for MGRS is essential for better prognosis. Generally, bortezomib-based chemotherapy is considered to be the firstline treatment strategy for MGRS due to the rapid and deep response [6, 21-23]. However, the benefit of the chemotherapy has been debated in the context of inconclusive bone marrow findings, especially when MIg is hardly detected in the serum or urine [24]; the requirement for chemotherapy needs to be explored further. ASCT also represents an important treatment option for MGRS [25-27]. A controversial regimen of renal transplantation, which is associated with a high rate of recurrence and graft loss, has also been used [2, 5, 28, 29]. Therapeutic strategies for MGRS should focus on the eradication of pathogenic clonal cells. However, the development of a clone-specific approach presents the considerable challenge of choosing the most effective and clone-specific treatment with the safest adverse event profile, particularly when no B cell or plasma cell clone has been detected $[4,30]$. Notably, the International Kidney and Monoclonal Gammopathy Research Group summarized specific therapeutic regimens according to MGRS disorder type. Options for LCDD treatment should take into account CKD stage and the availability of renal transplantation [31].

Overall patient survival at 1 and 5 years ( 89 and 70\%, respectively) is better than renal survival (67 and 17\%, respectively) [1, 5, 18, 31, 32]. Prognostic factors for MGRS include age, serum creatinine in primary diagnosis, presence of plasma cell myeloma, and extrarenal LC deposition [7, 33]. Lower initial serum creatinine is recognized as the most valuable prognostic indicator of renal survival in multivariate analysis [7, 34-36]. Two retrospective studies by Nasr et al. [35] and Pozzi et al. [36] showed that earlier detection and the availability of newer therapeutic regimens (such as bortezomib-based chemotherapy and ASCT), respectively, could reduce mortality in patients and improve MGRS prognosis and outcomes (Table 1). In addition, in 2015, committee members of the National Amyloidosis Centre highlighted the relationship between hematologic response and renal outcome in patients with MGRS. The rate of native GFR loss among patients who did not achieve either CR or VGPR with chemotherapy was $6.5 \mathrm{~mL} / \mathrm{min} /$ year, whereas the rate for those who achieved CR or VGPR improved by $6.1 \mathrm{~mL} / \mathrm{min} /$ year. The data also demonstrated that ASCT could be successful, even in patients with a low GFR. Surprisingly, that research was the first to indicate that there 
Table 1. The prognosis and outcomes of patients with MGRS based on different treatment

\begin{tabular}{lcc}
\hline & Pozzi et al. [36] & Nasr et al. [35] \\
\hline Time, years & $1978-2002$ & $1992-2011$ \\
Patients, $n$ & 63 & 64 \\
Mean duration of follow-up, months & 28 & 34 \\
No treatment & $14 \%$ & $14 \%$ \\
Chemotherapy & $78 \%$ & $57 \%$ \\
Bortezomib-based chemotherapy & $0 \%$ & $37.5 \%$ \\
ASCT & $8 \%$ & $29 \%$ \\
Reached ESRD & $57 \%$ & $39 \%$ \\
Died & $59 \%$ & $32 \%$ \\
Mean renal survival, months & 32 & 64 \\
Mean patient survival, months & 64 & 90 \\
\hline
\end{tabular}

was no statistical difference in the renal survival of MGRS patients with or without nephroticrange proteinuria $(>3 \mathrm{~g} / 24 \mathrm{~h}$ ). Furthermore, renal survival was not influenced by the degree of bone marrow plasmacytosis [8].

\section{Discussion/Conclusion}

The case we reported, of a patient diagnosed with IgA- $\lambda$ PLCDD, was rare and notable. Typically, the ratio of $\kappa$ to $\lambda$ is 9:1 in LCDD and abundant $\kappa$ in the serum is associated with LCDD, whereas abundant $\lambda$ is associated with AL amyloidosis [37]. In addition, IgG and IgM predominate in LCDD, and the percentage of LCDD associated with MM or lymphoproliferative disorders is over $80 \%[38,39]$. Thus, the probability of IgA- $\lambda$ PLCDD is slim; the reason for its occurrence - genetics, environment, or ethnicity - is a puzzling question. Furthermore, the question of links between LC types ( $\kappa$ or $\lambda$ ) and the specific disorders of MGRS (such as LCDD) remains open. The mechanism driving IgA- $\lambda$ PLCDD remains unknown.

In particular, the pathologic and morphologic features observed in the renal biopsy of this patient differed from the classic manifestations of LCDD. In classical LCDD, IF presents LCs as diffuse linear deposits along the GBM and TBM, and EM displays punctate, powdery, electron-dense deposits along the inner aspect of the GBM and the outer aspect of the TBM $[9,40,41]$. However, in this case, there was strong IF staining for $\lambda$-LCs along the glomerular capillary walls, TBM, and tubulointerstitium. Furthermore, the basement membrane was destroyed by EM, which could hardly distinguish the locations of the deposits. In addition, the subendothelial area was expanded with unknown material. Immunoelectron microscopy may have been needed to confirm the identity of the material and provide a definite diagnosis. Therefore, the diagnosis of LCDD in this patient required deliberation after the initial testing. These morphologic or histologic indices - glomerular density, number of tubules per glomerulus, tubular density, and fractal dimension of the tubular lumen - all have excellent correlation with the eGFR (estimated by CKD-EPI formula) [42] and are of utmost importance for patient prognosis.

Bortezomib can inhibit the progression of glomerulosclerosis and reduce proteinuria by interrupting the activation cascade of signaling pathways such as NF- $\kappa \mathrm{B}$ that lead to rapid renal deterioration [43]. Thus, bortezomib-based chemotherapy had been regarded as the first-line treatment for MGRS. Beyond that, ASCT can provide a better prognosis for patients. Bortezomib is ideally suited for incorporation into induction therapy before ASCT to improve 


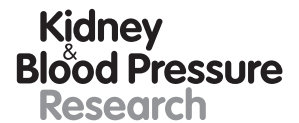

Kidney
Blood Pressure
Research

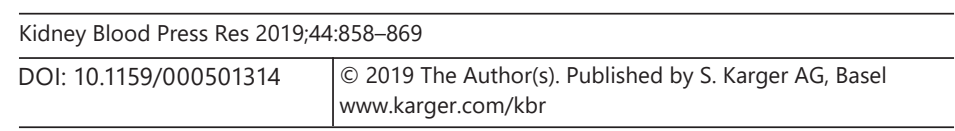

Huang et al.: Bortezomib/Autologous Transplant for Monoclonal Gammopathy of Renal Significance

efficacy [44]. However, there is a lack of large-scale analysis of the curative effects of bortezomib-based chemotherapy combined with ASCT, which must be urgently addressed. Despite this, this newer therapy regimen has been adopted clinically with good response rates [45-48]. Our patient accepted this regimen to achieve a better long-term outcome regardless of the financial burden. As expected, the regimen appeared successful.

LCs are filtered by the glomeruli, bound by LC receptors on the surface of mesangial cells and vessel smooth muscle cells, reabsorbed in proximal tubules by receptor-mediated endocytosis, and degraded in tubular cells by lysosomal enzymes [8, 9, 39, 49]. Thus, the kidney is the principal target for LC deposition. However, LCs can also deposit in other tissues, including brain, heart, lung, liver, spleen, pancreas, muscle, pharynx, cervical lymph nodes, gastrointestinal tract, and nervous system, resulting in varying degrees of organ dysfunction that are rarely symptomatic [50]. In this patient, there were no signs of extrarenal organ involvement, which may improve his prognosis and outcome. His diagnosis of PLCDD that was not secondary to MM also improved his prognosis over patients with MM. In addition, the patient came to hospital early without serious kidney involvement, which protected him from the rapid loss of renal function. Due to the active treatment with chemotherapy and ASCT, he achieved hematologic CR and renal improvement. Moreover, he maintained hematologic CR for a relatively long time after ASCT. Thus, the successful treatment of the patient with bortezomibbased chemotherapy and ASCT may shed some light on the treatment of MGRS.

We reported this special case to enhance awareness of MGRS and describe options for a therapeutic regimen for MGRS. However, the case is complicated by some problems. The diagnosis of this patient with PLCDD was not clear and definite. The follow-up observation period was short (only 6 months), which may not completely reflect the curative effect of the treatment, and the renal survival and patient survival were not determined. The effect of the nature of the LC $(\operatorname{IgA}-\lambda)$ on the efficacy of the treatment or prognosis is unknown. In addition, it seems that ASCT actually worsened the clinical scenario (in terms of creatinine, eGFR, hemoglobin, albumin, and proteinuria). Although these changes were transient, they may have resulted from a posttransplant reaction. There is no indication that the ASCT was the reason for the improvement over the long-term.

MGRS is associated with a wide spectrum of kidney diseases, which are classified by the type, size, localization, and organization of deposited MIg in the absence of hematologic malignancy or other myeloma-defining events. Renal biopsy (especially for IF and EM) should be performed when MGRS is highly suspected. The treatment of MGRS is mainly based on chemotherapy that should be adapted to the lymphocytic or plasmacytic nature of the underlying clone, renal function based on CKD stage, and the presence or absence of extrarenal involvement. Bortezomib-based chemotherapy combined with ASCT may be a prospective treatment of MGRS, but the effects of ASCT remains unclear due to the complicated settings. In addition, the collateral effects of this therapy must be further investigated and taken into consideration. The therapeutic goal is to achieve at least VGPR and to stabilize or improve renal function.

Despite the rarity of the entities, randomized trials should be designed to identify the most beneficial and effective therapeutic approaches for affected patients. As MGRS is a heterogeneous and relatively rare entity, cooperation between nephrologists and hematologists in well-designed prospective collaborative studies is required to improve management. The development of methods to reduce the serious side effects of chemotherapy drugs and improve patient prognosis is a noteworthy challenge that should be discussed and investigated. 


\section{Kidney \\ Blood Pressure \\ Research}

\begin{tabular}{l|l}
\hline Kidney Blood Press Res 2019;44:858-869 \\
\hline DOI: 10.1159/000501314 & $\begin{array}{l}\text { ○ 2019 The Author(s). Published by S. Karger AG, Basel } \\
\text { www.karger.com/kbr }\end{array}$ \\
\hline
\end{tabular}

Huang et al.: Bortezomib/Autologous Transplant for Monoclonal Gammopathy of Renal Significance

\section{Acknowledgement}

We are thankful to the patient for his participation in the study. This work was supported by grants from the National Natural Science Foundation of China (No. 81770736 and No. 30800532), the Doctoral Fund of the Ministry of Education of China (No. 200804871122), the Natural Science Foundation of Hubei Province (No. 2015CFB467 and No. 2014CFB197), the Chinese Medical Association (No. 14050510588), the Huazhong University of Science and Technology (No. 2015LC033 and No. 2015QN198).

\section{Statement of Ethics}

Written informed consent was obtained from the patient prior to his inclusion in the study.

\section{Disclosure Statement}

The authors declare that the research was conducted in the absence of any commercial or financial relationships that could be construed as a potential conflict of interest.

\section{Author Contributions}

J.X. designed and led this study. J.H. searched the literature and drafted the manuscript. C.Y.S., H.S., and C.Z. analyzed the data. J.X. revised the manuscript. All authors reviewed the manuscript.

\section{References}

1 Leung N, Bridoux F, Hutchison CA, Nasr SH, Cockwell P, Fermand JP, et al.; International Kidney and Monoclonal Gammopathy Research Group. Monoclonal gammopathy of renal significance: when MGUS is no longer undetermined or insignificant. Blood. 2012 Nov;120(22):4292-5.

2 Bridoux F, Leung N, Hutchison CA, Touchard G, Sethi S, Fermand JP, et al.; International Kidney and Monoclonal Gammopathy Research Group. Diagnosis of monoclonal gammopathy of renal significance. Kidney Int. 2015 Apr;87(4):698-711.

3 Sethi S, Rajkumar SV, D'Agati VD. The Complexity and Heterogeneity of monoclonal immunoglobulin-associated renal diseases. J Am Soc Nephrol. 2018 Jul;29(7):1810-23.

4 Hogan JJ, Weiss BM. Bridging the Divide: An Onco-Nephrologic Approach to the Monoclonal Gammopathies of Renal Significance. Clin J Am Soc Nephrol. 2016 Sep;11(9):1681-91.

5 Ciocchini M, Arbelbide J, Musso CG. Monoclonal gammopathy of renal significance (MGRS): the characteristics and significance of a new meta-entity. Int Urol Nephrol. 2017 Dec;49(12):2171-5.

6 Cohen C, Javaugue V, Joly F, Arnulf B, Fermand JP, Jaccard A, et al. [Randall-type monoclonal immunoglobulin deposition disease: from diagnosis to treatment]. Nephrol Ther. 2016 Jun;12(3):131-9.

7 Lin J, Markowitz GS, Valeri AM, Kambham N, Sherman WH, Appel GB, et al. Renal monoclonal immunoglobulin deposition disease: the disease spectrum. J Am Soc Nephrol. 2001 Jul;12(7):1482-92.

8 Sayed RH, Wechalekar AD, Gilbertson JA, Bass P, Mahmood S, Sachchithanantham S, et al. Natural history and outcome of light chain deposition disease. Blood. 2015 Dec;126(26):2805-10.

9 Batuman V, Guan S. Receptor-mediated endocytosis of immunoglobulin light chains by renal proximal tubule cells. Am J Physiol. 1997 Apr;272(4 Pt 2):F521-30.

10 Kyle RA, Durie BG, Rajkumar SV, Landgren O, Blade J, Merlini G, et al.; International Myeloma Working Group. Monoclonal gammopathy of undetermined significance (MGUS) and smoldering (asymptomatic) multiple myeloma: IMWG consensus perspectives risk factors for progression and guidelines for monitoring and management. Leukemia. 2010 Jun;24(6):1121-7. 


\section{Kidney \\ Blood Pressure \\ Research}

\begin{tabular}{l|l}
\hline Kidney Blood Press Res 2019;44:858-869 \\
\hline DOI: 10.1159/000501314 & $\begin{array}{l}\text { @ 2019 The Author(s). Published by S. Karger AG, Basel } \\
\text { www.karger.com/kbr }\end{array}$ \\
\hline
\end{tabular}

Huang et al.: Bortezomib/Autologous Transplant for Monoclonal Gammopathy of Renal Significance

11 Palladini G, Dispenzieri A, Gertz MA, Kumar S, Wechalekar A, Hawkins PN, et al. New criteria for response to treatment in immunoglobulin light chain amyloidosis based on free light chain measurement and cardiac biomarkers: impact on survival outcomes. J Clin Oncol. 2012 Dec;30(36):4541-9.

12 Radhakrishnan J, Cattran DC. The KDIGO practice guideline on glomerulonephritis: reading between the (guide)lines-application to the individual patient. Kidney Int. 2012 Oct;82(8):840-56.

13 Sethi S, Fervenza FC, Rajkumar SV. Spectrum of manifestations of monoclonal gammopathy-associated renal lesions. Curr Opin Nephrol Hypertens. 2016 Mar;25(2):127-37.

14 Koo EH, Shin JH, Jang HR, Park HD, Kwon GY, Huh W, et al. Diagnostic performances of M-protein tests according to the clinical presentations of kidney disease. Eur J Intern Med. 2016 Sep;33:88-92.

15 Correia SO, Santos S, Malheiro J, Cabrita A, Martins S, Santos J. Monoclonal gammopathy of renal significance: diagnostic workup. World J Nephrol. 2017 Mar;6(2):72-8.

16 Willrich MA, Murray DL, Kyle RA. Laboratory testing for monoclonal gammopathies: focus on monoclonal gammopathy of undetermined significance and smoldering multiple myeloma. Clin Biochem. 2018 Jan;51: 38-47.

17 Leung N, Barnidge DR, Hutchison CA. Laboratory testing in monoclonal gammopathy of renal significance (MGRS). Clin Chem Lab Med. 2016 Jun;54(6):929-37.

18 Randall RE, Williamson WC Jr, Mullinax F, Tung MY, Still WJ. Manifestations of systemic light chain deposition. Am J Med. 1976 Feb;60(2):293-9.

19 Gavriatopoulou M, Musto P, Caers J, Merlini G, Kastritis E, van de Donk N, et al. European myeloma network recommendations on diagnosis and management of patients with rare plasma cell dyscrasias. Leukemia. 2018 Sep;32(9):1883-98.

20 Steiner N, Göbel G, Suchecki P, Prokop W, Neuwirt H, Gunsilius E. Monoclonal gammopathy of renal significance (MGRS) increases the risk for progression to multiple myeloma: an observational study of 2935 MGUS patients. Oncotarget. 2017 Dec;9(2):2344-56.

21 Minarik J, Scudla V, Tichy T, Pika T, Bacovsky J, Lochman P, et al. Induction treatment of light chain deposition disease with bortezomib: rapid hematological response with persistence of renal involvement. Leuk Lymphoma. 2012 Feb;53(2):330-1.

22 San-Miguel JF, Richardson PG, Sonneveld P, Schuster MW, Irwin D, Stadtmauer EA, et al. Efficacy and safety of bortezomib in patients with renal impairment: results from the APEX phase 3 study. Leukemia. $2008 \mathrm{Apr}$; 22(4):842-9.

23 Cohen C, Royer B, Javaugue V, Szalat R, El Karoui K, Caulier A, et al. Bortezomib produces high hematological response rates with prolonged renal survival in monoclonal immunoglobulin deposition disease. Kidney Int. 2015 Nov;88(5):1135-43.

24 Murray DL, Seningen JL, Dispenzieri A, Snyder MR, Kyle RA, Rajkumar SV, et al. Laboratory persistence and clinical progression of small monoclonal abnormalities. Am J Clin Pathol. 2012 Oct;138(4):609-13.

25 Heher EC, Goes NB, Spitzer TR, Raje NS, Humphreys BD, Anderson KC, et al. Kidney disease associated with plasma cell dyscrasias. Blood. 2010 Sep;116(9):1397-404.

26 Harousseau JL, Moreau P. Autologous hematopoietic stem-cell transplantation for multiple myeloma. N Engl J Med. 2009 Jun;360(25):2645-54.

27 Knudsen LM, Nielsen B, Gimsing P, Geisler C. Autologous stem cell transplantation in multiple myeloma: outcome in patients with renal failure. Eur J Haematol. 2005 Jul;75(1):27-33.

28 Giordano M, Santangelo L, Scarasciulli ML, Calvario A, Miragliotta G, Giordano P, et al. Monoclonal gammopathy of undetermined significance in pediatric kidney transplant: a possible role of Epstein-Barr virus. Pediatr Transplant. 2014 Feb;18(1):42-6.

29 Nambirajan A, Bhowmik D, Singh G, Agarwal SK, Dinda AK. Monoclonal gammopathy of renal significance with light-chain deposition disease diagnosed postrenal transplant: a diagnostic and therapeutic challenge. Transpl Int. 2015 Mar;28(3):375-9.

30 Rosner MH, Edeani A, Yanagita M, Glezerman IG, Leung N; American Society of Nephrology Onco-Nephrology Forum. Paraprotein-Related Kidney Disease: Diagnosing and Treating Monoclonal Gammopathy of Renal Significance. Clin J Am Soc Nephrol. 2016 Dec;11(12):2280-7.

31 Fermand JP, Bridoux F, Kyle RA, Kastritis E, Weiss BM, Cook MA, et al.; International Kidney and Monoclonal Gammopathy Research Group. How I treat monoclonal gammopathy of renal significance (MGRS). Blood. 2013 Nov;122(22):3583-90.

32 Glavey SV, Leung N. Monoclonal gammopathy: the good, the bad and the ugly. Blood Rev. 2016 May;30(3): 223-31.

33 Chauvet S, Frémeaux-Bacchi V, Petitprez F, Karras A, Daniel L, Burtey S, et al. Treatment of B-cell disorder improves renal outcome of patients with monoclonal gammopathy-associated C3 glomerulopathy. Blood. 2017 Mar;129(11):1437-47.

34 Li XM, Rui HC, Liang DD, Xu F, Liang SS, Zhu XD, et al. Clinicopathological characteristics and outcomes of light chain deposition disease: an analysis of 48 patients in a single Chinese center. Ann Hematol. 2016 May;95(6): 901-9.

35 Nasr SH, Valeri AM, Cornell LD, Fidler ME, Sethi S, D’Agati VD, et al. Renal monoclonal immunoglobulin deposition disease: a report of 64 patients from a single institution. Clin J Am Soc Nephrol. 2012 Feb;7(2):231-9.

36 Pozzi C, D’Amico M, Fogazzi GB, Curioni S, Ferrario F, Pasquali S, et al. Light chain deposition disease with renal involvement: clinical characteristics and prognostic factors. Am J Kidney Dis. 2003 Dec;42(6):1154-63. 
37 Gertz MA. Managing light chain deposition disease. Leuk Lymphoma. 2012 Feb;53(2):183-4.

38 van de Donk NW, Palumbo A, Johnsen HE, Engelhardt M, Gay F, Gregersen H, et al.; European Myeloma Network. The clinical relevance and management of monoclonal gammopathy of undetermined significance and related disorders: recommendations from the European Myeloma Network. Haematologica. 2014 Jun; 99(6): 984-96.

39 Ronco P, Plaisier E, Mougenot B, Aucouturier P. Immunoglobulin light (heavy)-chain deposition disease: from molecular medicine to pathophysiology-driven therapy. Clin J Am Soc Nephrol. 2006 Nov;1(6):1342-50.

40 Jimenez-Zepeda VH. Light chain deposition disease: novel biological insights and treatment advances. Int J Lab Hematol. 2012 Aug;34(4):347-55.

41 Buxbaum J, Gallo G. Nonamyloidotic monoclonal immunoglobulin deposition disease. Light-chain, heavychain, and light- and heavy-chain deposition diseases. Hematol Oncol Clin North Am. 1999 Dec;13(6):123548.

42 Nigro M, Viggiano D, Ragone V, Trabace T, di Palma A, Rossini M, et al. A cross-sectional study on the relationship between hematological data and quantitative morphological indices from kidney biopsies in different glomerular diseases. BMC Nephrol. 2018 Mar;19(1):62.

43 Gandolfi S, Laubach JP, Hideshima T, Chauhan D, Anderson KC, Richardson PG. The proteasome and proteasome inhibitors in multiple myeloma. Cancer Metastasis Rev. 2017 Dec;36(4):561-84.

44 Leung N, Drosou ME, Nasr SH. Dysproteinemias and glomerular disease. Clin J Am Soc Nephrol. 2018 Jan; 13(1):128-39.

45 Lorenz EC, Gertz MA, Fervenza FC, Dispenzieri A, Lacy MQ, Hayman SR, et al. Long-term outcome of autologous stem cell transplantation in light chain deposition disease. Nephrol Dial Transplant. 2008 Jun;23(6):2052-7.

46 Weichman K, Dember LM, Prokaeva T, Wright DG, Quillen K, Rosenzweig M, et al. Clinical and molecular characteristics of patients with non-amyloid light chain deposition disorders, and outcome following treatment with high-dose melphalan and autologous stem cell transplantation. Bone Marrow Transplant. 2006 Sep; 38(5):339-43.

47 Jimenez-Zepeda VH, Trudel S, Winter A, Reece DE, Chen C, Kukreti V. Autologous stem cell transplant for light chain deposition disease: incorporating bortezomib to the induction therapy. Am J Hematol. 2012 Aug;87(8): 822-3.

48 Tovar N, Cibeira MT, Rosiñol L, Solé M, de Larrea CF, Escoda L, et al. Bortezomib/dexamethasone followed by autologous stem cell transplantation as front line treatment for light-chain deposition disease. Eur J Haematol. 2012 Oct;89(4):340-4.

49 Pozzi C, Locatelli F. Kidney and liver involvement in monoclonal light chain disorders. Semin Nephrol. 2002 Jul;22(4):319-30.

50 Kim HJ, Park E, Lee TJ, Do JH, Cha YJ, Lee SJ. A case of isolated light chain deposition disease in the duodenum. J Korean Med Sci. 2012 Feb;27(2):207-10. 